\title{
EL ENIGMA DE LO DIONISÍACO Y LA INTENCIÓN DE EL NACIMIENTO DE LA TRAGEDIA
}

The enigma of the Dionysian and the intention in The Birth of Tragedy

\author{
Patrick Wotling
}

Universidad de Reims

«Lo dionisíaco aparece aquí como el poder artístico eterno y original»1

RESUMEN: Este estudio se fija en las dificultades que rodean el estatus exacto de la noción de «dionisíaco » en El nacimiento de la tragedia, así como en la naturaleza de las relaciones entre pulsión dionisíaca y pulsión apolínea. Si bien se presentan inicialmente bajo el modo del dualismo, indicios importantes sugieren al examinarlos que su situación es disimétrica, lo dionisíaco asumiendo el papel de fundamento del proceso apolíneo. Pero a esto se añade que lo dionisiaco, sin embargo, llega a ser pensable y accesible a partir de una lógica apolínea. Nietzsche, a través de una lógica compleja que pone en práctica, ¿no buscará ante todo en El nacimiento de la tragedia revelarnos la especificidad de nuestra relación con la realidad?

Palabras clave: dionisíaco - apolíneo - tragedia - realidad

ABSTRACT: his paper investigates the status and difficulties of the «dionysian» in The Birth of Tragedy, as well as the nature of the link between the apollinian and the dionysian drives. Although they are originally introduced in keeping with a dualistic perspective, important clues point to the possibility that they might in fact be asymmetrical, and suggest that the dionysian is to be considered the basis underlying the apollinian process. At the same time, however, it appears that the dionysian can only be thought of and reached by means of the apollinian process. What, then, does Nietzsche exactly aim at when developing the complicated logic of The Birth of Tragedy? Is it not, first and foremost, to reveal the peculiarity of our relationship to reality?

Keywords: dyonisian - apollinian - tragedy - reality

\section{EL PROBLEMA PLANTEADO POR LA PAREJA APOLÍNEO/DIONISÍACO}

Se ha convertido en una costumbre bastante corriente, en el seno de los comentaristas nietzscheanos, considerar como de suyo que se produce una ruptura en el pensamiento del filósofo después de El nacimiento de la tragedia. Esta visión de las cosas se debe en parte al carácter bastante atípico de las temáticas puestas en juego por esa primera obra, que ya no volveremos a encontrar por lo general en los textos posteriores; pero es sobre todo la reivindicación de una posición de Nietzsche que se designa como metafísica —incluso si se trata de una «metafísica

1. El nacimiento de la tragedia/GT, $\mathbb{\$} 25$, OC I 267-268. El subrayado de la parte central es nuestro. 
del arte» ${ }^{2}$, de una «metafísica estética» ${ }^{3}$, o de una «metafísica de artista ${ }^{4}$, y no la o las, ante todo ontológicas, que ha o han ocupado el lugar central de la escena en la historia de la filosofía - lo que ha fascinado a numerosos comentaristas, y es la presencia de este elemento lo que ha pesado de manera preponderante para justificar a sus ojos la convicción de que se producirá también en él una mutación profunda después de este primer texto. Una lectura semejante, por lo demás, parece que se confirma con las declaraciones del mismo Nietzsche. ¿No proclama él en la época de la redacción de Humano, demasiado humano: «A los lectores de mis escritos precedentes quiero manifestar de forma expresa que he abandonado los puntos de vista metafísico-artísticos que en esencia dominaban en ellos; son agradables, pero insostenibles. Quien se permite hablar en público en fecha temprana, por lo habitual se ve obligado al poco tiempo a contradecirse públicamente» ${ }^{5}$. Este aviso a los lectores no es ciertamente el único entre borradores y escritos no publicados. Pero es tan completo y tan solemne que impresiona. Por consiguiente, ¿̇no merece que se lo coloque en un lugar destacado del escenario?

¿Pero es este realmente un apoyo suficiente? ¿La realidad del giro está suficientemente atestiguada por la reivindicación no publicada de una inflexión de la posición doctrinal sobre la cuestión de la relación con la metafísica? Y si lo está, ¿dicha reivindicación indica de manera luminosa el contenido preciso de lo que es abandonado? Inevitablemente hay puntos débiles que amenazan una interpretación tan equívoca. Pues, por una parte, podría darse una cierta confusión con la comprensión que los comentaristas hacen ordinariamente del sentido del término «metafísica», del que hace uso Nietzsche en sus primeros textos; y por otra parte, será perfectamente plausible que la novedad aportada por la reflexión nietzscheana sobre el terreno filosófico no haya querido adoptar tesis particulares, «metafísicas» o no metafísicas, sino cambiar profundamente la manera de reflexionar y de conducir el pensamiento.

Estamos de acuerdo por esta razón con el análisis perspicaz de James Porter, quien sostiene por su parte la tesis siguiente: «El intento de parcelar la carrera de Nietzsche en períodos o fases según su supuesta aceptación o rechazo de principios metafísicos está condenado al fracaso» ${ }^{6}$. La ruptura, en estas condiciones, si se quiere encontrar una, se habría consumado más bien en El nacimiento de la tragedia mismo, en relación con el universo filosófico anterior, que en las obras posteriores al mencionado libro de El nacimiento de la tragedia, en relación con este último. En resumen, una continuidad real no es inviable en el seno del pensamiento nietzscheano; pero quizás solo aparece si se elabora una comprensión de la filosofía que va más allá de la que pretende caracterizarla exclusivamente por

2. El nacimiento de la tragedia, $\mathbb{\$} 24$.

3. Ibid., $\mathbb{} 5$.

4. Ibid., "Ensayo de autocrítica», $\mathbb{2}$. Cualquiera que sea la cualificación más adaptada, la dirección del análisis contemplado por esta posición es explícitamente formulada desde las primeras líneas de la obra de 1872: el arte constituye la meta suprema y la actividad propiamente metafísica de esta vida» (El nacimiento de la tragedia, «Prólogo a Richard Wagner»).

5. FP I 361, 23[159].

6. J. I. Porter, The Invention of Dionysus. An Essay on The Birth of Tragedy, Stanford, Stanford UP, 2000, p. 8. Véase así mismo la siguiente noción muy importante: «El deseo de periodizar a Nietzsche dentro de marcos fijos ciega a los lectores en relación con los gestos complejos y autocontradictorios que recorren todos sus escritos» (ibid.) 
un corpus de doctrinas y de tesis. Ya hemos llamado la atención sobre la importancia de esta perspectiva para quien quiere tomar en su especificidad la lógica de la intervención de Nietzsche sobre el terreno filosófico ${ }^{7}$ : conviene superar el diálogo doctrinal en provecho del diálogo problemático. Nada asegura, en estas condiciones, que el cambio de posición relativo a la metafísica de artista testimonie la emergencia de un segundo Nietzsche, en el sentido en el que se puede hablar, por ejemplo, de un segundo Wittgenstein. Esto se debe a que la intervención de Nietzsche se centra más bien en los esquemas de pensamiento, y en particular en los esquemas de pensamiento ilegítimos, que constituyen los componentes mismos del análisis filosófico, que en la recusación de contenidos doctrinales, siempre segundos y siempre condicionados por ciertos esquemas de pensamiento particulares. El planteamiento de la cuestión de la continuidad o discontinuidad entre la primera obra publicada por el filósofo y sus libros posteriores hay que aclararlo en otro contexto. Y es lo que nos parece justamente indicar el matiz, capital, aportado por el Ensayo de autocrítica, de 1866, al condenar retrospectivamente la metafísica de artista: «a toda esta metafísica-de-artista se la puede denominar arbitraria, ociosa, fantástica -, lo esencial al respecto es que tal metafísica delata ya un espiritu que alguna vez, asumiendo todos los riesgos, se defenderá contra la interpretación moral y el significado moral de la existencia» ${ }^{8}$.

¿Pone de manifiesto el análisis de los esquemas de pensamiento aplicados por El nacimiento de la tragedia una afinidad que se identifica con los esquemas sobre los que se apoyará la reflexión de los años siguientes, hasta 1888? Tal es el tipo de investigación que debe ser realizada. En un estudio reciente, nos hemos esforzado en releer y plantearnos algunas cuestiones sobre la primera obra de Nietzsche ${ }^{9}$. Del mismo modo, nos gustaría abordar aquí uno de los aspectos de esta amplia cuestión: el que concierne a la manera en la que Nietzsche piensa exactamente la naturaleza de la relación entre lo apolíneo y lo dionisíaco en esa misma obra. Esta cuestión no ha sido fácil de resolver en la lectura y sobre todo en la relectura de este primer libro. Pero posee una importancia primordial para la comprensión de la naturaleza de la empresa nietzscheana. Y tratándose de la cuestión de la unidad o de la ruptura eventual, en el seno del desarrollo del pensamiento del filósofo, es completamente decisiva. Recordemos, en efecto, que uno de los esquemas de pensamiento característico de la reflexión nietzscheana de la década de 1880 es el de la total homogeneidad de lo real. Nietzsche rechaza sin equívocos toda idea de una diferencia de naturaleza entre diferentes estratos de la realidad. Cualquiera que sea su forma, una ontología diferenciada es filológicamente inaceptable; por lo tanto también es el caso para la ontología en general, puesto que lo que tiene realidad, lo que solo tiene realidad, son los procesos interpretativos que Nietzsche designa con una serie variada de términos de los que los más frecuentes son «pul-

7. Véase en particular nuestro estudio «La culture comme problème», en «Oui, l'homme fut un essai». La philosophie de l'avenir selon Nietzsche, Paris : PUF, 2016.

8. El nacimiento de la tragedia, «Ensayo de autocrítica», $\$ 5$, OC I 333. Ponemos en cursiva el término "espíritu», en torno al cual se articulan el matiz decisivo de la declaración de Nietzsche y la limitación de la condena que pronuncia.

9. P. Wotling «'Mon premier renversement de toutes les valeurs'. La naissance de la tragédie et la mise en place des invariants du questionnement nietzschéen», en Nietzsche. Les premiers textes sur les Grecs, Reims: Editions et presses de l'université de Reims (Épure), 2016, pp. 139-169. 
siones», «instintos», «afectos», o «interpretaciones». En resumen, las voluntades de poder - término que sin embargo a Nietzsche le desagrada emplear en plural一. ¿No habría justamente una clara ruptura en relación al modo de pensamiento de El nacimiento de la tragedia, que parece defender un dualismo pulsional fundamental, separando irremediablemente lo dionisíaco y lo apolíneo?

\section{2. ¿LO DIONISÍACO, FUNDAMENTO DE LO APOLÍNEO?}

Retomemos los puntos esenciales y bien conocidos del debate. Los primeros parágrafos de El nacimiento de la tragedia se proponen introducir dos elementos, designados desde la primera frase del libro como «lo apolíneo» y «lo dionisíaco», elementos a partir de los cuales se construirá luego todo el desarrollo de la obra, hasta su parágrafo conclusivo. Estos elementos son caracterizados como «pulsiones de la naturaleza», y su carácter concreto es luego explicitado a partir del modelo que proporcionan dos fenómenos fisiológicos distintos, el sueño y la embriaguez. Al comienzo de la obra, estos dos procesos artísticos son claramente presentados no solamente como distintos, sino incluso como estrictamente antagónicos, tal y como lo subraya explícitamente la explotación de los modelos fisiológicos: «fenómenos fisiológicos entre los que se puede constatar una antítesis que se corresponde con la existente entre lo apolíneo y lo dionisíaco» ${ }^{10}$. Por consiguiente, Nietzsche parece que defiende en 1872 una comprensión diversificada, no homogénea, de la realidad.

El destino de estas dos nociones, como se sabe, no limita su utilización únicamente a El nacimiento de la tragedia. Juntas, o la mayoría de las veces separadas (en ese caso, con una frecuencia superior para la noción de lo dionisíaco), resurgirán años más tarde en los escritos de Nietzsche, tanto en los póstumos como en los publicados. Entre estos, un texto, Crepúsculo de los ídolos, posee un interés especialmente particular: es el primero que después de diez años vuelve a la confrontación de estas dos nociones. Así pues, y de una manera totalmente llamativa, en lo sucesivo ya no se defenderá su antagonismo, sino la aproximación de las dos pulsiones. $\mathrm{Su}$ acercamiento es en efecto tan extremadamente claro que llega a proclamar la identidad de naturaleza de lo apolíneo y lo dionisíaco, puesto que el Crepúsculo de los ídolos retoma la modelización fisiológica utilizada en El nacimiento de la tragedia, pero para reducir esta vez conjuntamente los dos tipos de procesos a un solo y único fenómeno, el de la embriaguez: «¿̨ué significa la antítesis conceptual, introducida por mí en la estética, de lo apolíneo y lo dionisíaco, entendidos ambos como especies de embriaguez?»11. Es en el seno de la embriaguez donde, de manera derivada, secundariamente, sin importancia al fin, puede encontrarse una diferencia. Nietzsche precisa entonces la particularidad de cada una de estas dos formas de embriaguez, una que es especializada y se restringe al aparato visual, la otra, en cambio, es general: «La embriaguez apolínea mantiene excitado sobre todo el ojo, de manera que este adquiere la fuerza de producir una visión. El pintor, el artista plástico, el poeta épico son visionarios par excellence. En el estado dionisíaco, por el contrario, el sistema total de los afectos es el que está excitado e intensificado: de modo que este sistema descarga de una vez todos sus medios de expresión y

10. El nacimiento de la tragedia, $\mathbb{S} 1$. Subrayamos el término «antítesis». OC I 338.

11. Crepúsculo de los ídolos, «Incursiones de un intempestivo», $\mathbb{S} 10$. OC IV 660. 
al mismo tiempo hace que se exteriorice la fuerza que es capaz de representar, de imitar, de transfigurar, de transformar, que se manifieste todo género de mímica y de arte dramático» ${ }^{12}$.

Lo que sorprende es la afirmación inicial. Como se acaba de mencionar, en El nacimiento de la tragedia, Nietzsche se refería a dos modelos fisiológicos heterogéneos para presentar los dos procesos, los cuales por tanto no eran en modo alguno entendidos como «dos especies de embriaguez». Pero paralelamente, uno no puede evitar, leyendo el libro de 1872, el sentimiento extraño de que la distancia que separa a las dos pulsiones parece admitir variaciones importantes según las secuencias del análisis y los contextos en los que ellas están involucradas.

Si a primera vista la situación carece de claridad a lo largo de todo El nacimiento de la tragedia, donde parece hacer coexistir la tesis de la diferencia de naturaleza y una serie de indicios o de fórmulas que sugieren, al contrario, la afinidad esencial de las dos pulsiones, una pieza última viene a modificar totalmente el asunto: se trata del último parágrafo de El nacimiento de la tragedia. En la segunda parte de la obra, que comienza en el $\$ 16$, Nietzsche ha estudiado sucesivamente el antagonismo entre arte y ciencia, luego el triunfo del espíritu socrático en la cultura europea moderna, y las debilidades y peligros de esa forma de cultura que valora exclusivamente el conocimiento, antes de pasar al poder salvador del arte trágico, apoyado en el mito, y en la esperanza de un resurgimiento de la cultura trágica en la época contemporánea. Acaba de explicar la elucidación del estatus del mito trágico a partir del análisis del fenómeno de la disonancia, y, con el círculo ya cerrado, el breve $\$ 25$ no tiene otra función que la de proporcionar una conclusión general a la larga y compleja serie de investigaciones llevadas a cabo por la obra. Así pues, si desempeña su papel de conclusión, lo hace aportando no simplemente una recapitulación, sino nada menos que una inflexión mayor en las tesis defendidas previamente: lo dionisíaco ya no se yuxtapone a lo apolíneo, sino que habla, esta vez, de «un dominio artístico situado más allá de lo apolíneo». ¿Cómo se ha de entender el significado de este «más allá»? Más bien como un cambio radical de posición que en adelante se constata. La continuación del texto lo muestra sin ambigüedad: a lo dionisíaco se le atribuye ya una posición claramente privilegiada, principal, fundadora, y es calificado ya de «violencia artística eterna y originaria», un poder que resulta estar en el origen del conjunto del mundo fenoménico: «Aquí lo dionisíaco comparado con lo apolíneo se muestra como la violencia artística eterna y originaria que proporciona existencia al mundo entero del fenómeno: en cuyo centro se hace necesaria una nueva apariencia de transfiguración, para mantener con vida el animado mundo de la individuación ${ }^{13}$. Siguiendo una especie de crescendo, el parágrafo llega hasta presentar, en conclusión, lo dionisiaco como la raíz misma de toda existencia, usando un término que Nietzsche evita generalmente emplear por pertenecer al pensamiento idealista: «Sin embargo, del fundamento de toda existencia, del subsuelo dionisíaco del mundo solo es lícito que introduzca en la conciencia del individuo humano justo aquella parte que puede ser superada de nuevo por esa fuerza apolínea...» ${ }^{14}$. Tal y como nos muestra este capítulo conclusivo, la esfera de lo apolíneo ya no se considera más de una forma absoluta como

12. Ibid.

13. El nacimiento de la tragedia, $\$ 25$. La parte central subrayada por nosotros.

14. Ibid., subrayamos la palabra «subsuelo». 
una esfera autónoma; ahora se presenta como enteramente subordinada a la de lo dionisíaco, de la que es descrita como su producto.

$\mathrm{Si}$, sobre la base de esta sorprendente afirmación final, que pone en tela de juicio las certezas, se procede a una relectura crítica de El nacimiento de la tragedia, se confirma el sentimiento que señalábamos más arriba: de hecho, la distancia entre las dos pulsiones admite según los casos variaciones, es decir, sobre todo aminoraciones, y en las principales secuencias del análisis que Nietzsche consagra al proceso apolíneo, se ocupa de insertar aberturas que sugieren una relación distinta que la de alteridad. De este modo, al comienzo del capítulo 17 insiste sobre la unidad de un enfoque común en el arte dionisíaco y el arte apolíneo: a saber, favorecer el compromiso de afirmación de la vida, o como dice a veces Nietzsche, convencer del placer de la existencia. Pero mucho antes, desde los primeros capítulos de la obra, esa tendencia está discretamente en ella: el célebre episodio de Midas y de la persecución de Sileno, en el capítulo 3, poseía ya una dimensión un poco enigmática en la medida en que parecía implicar que lo apolíneo solo puede surgir de un fondo dionisíaco ${ }^{15}$, instaurando una diferencia en relación a la presentación de aspecto dualista de las primera líneas de El nacimiento de la tragedia. El final del capítulo 2, que desarrolla el análisis de la lógica apolínea, dando a entender que podría existir una identidad subterránea entre las dos pulsiones mientras que proclamaba que el griego apolíneo experimentaba frente al mundo de lo dionisíaco el sentimiento sordo de que este último no le era extraño ${ }^{16}$. El capítulo 4 iba en la misma dirección, afirmando esta vez de una manera más explícita que este griego apolíneo estaba íntimamente ligado a los Titanes y héroes derrocados del universo dionisíaco arcaico. Por consiguiente, se puede afirmar que si el texto produce en el lector la sensación de que el análisis parte de una dualidad real, de una división natural entre estas dos pulsiones ${ }^{17}$, todo parece indicar que esta sensación se desvanece gradualmente a medida que se progresa en la construcción presentada por la obra, para dejar finalmente un lugar a la convicción de que lo dionisíaco, lejos de ser simplemente el hermano enemigo de lo apolíneo, posee una prioridad, una prioridad tal que le confiere incluso un estatus de universalidad, el de fundamento de toda realidad - lo que designan, quizás, las fórmulas inesperadas bajo la pluma de Nietzsche de «un originario» o a veces de «ser originario». ¿Es en este sentido en el que habría que hablar, por consiguiente, de «metafísica» para situar la posición de esta primera obra de Nietzsche? Si este último defiende finalmente la idea de que lo dionisiaco constituye el principio de toda realidad, de hecho, por la estructura de pensamiento que esto implica, estaríamos en una situación clásicamente metafísica, en el sentido más tradicional del término.

Es el momento de subrayar un matiz capital. A saber, el hecho de que este principio o fundamento, si efectivamente es uno, se parece muy poco al tipo de objeto que permite clásicamente pensar la idea de principio o la idea de fundamento. $\mathrm{Y}$ esto porque este «fundamento de toda existencia» debe ser caracterizado a

15. Lo que confirmará claramente el análisis del proceso de transfiguración apolínea en el capítulo 4.

16. " «... todo aquello no le era ciertamente tan extraño», «su conciencia apolínea le ocultaba ese mundo dionisíaco solo como un velo» (El nacimiento de la tragedia, $\$ 2$, OC I 344).

17. El nacimiento de la tragedia, $\mathbb{\$} 1$. 
partir del esquema de la contradicción, y ciertamente no por la inmutabilidad, la plenitud y el reposo en la identidad de sí que son usualmente las determinaciones esenciales del ser. Las designaciones de apariencia metafísica que uno se encuentra en El nacimiento de la tragedia tienen aquí, como siempre, algo de engañoso. Este «subsuelo [...] del mundo» que se ve, a veces calificado de unidad, es simultáneamente atravesado por la contradicción que constituye su propia esencia. Conforme al pensamiento que sugiere la figura de Dioniso lacerado, Nietzsche piensa desde esta época lo real como desunión y conflicto. Los textos preparatorios a los escritos sobre los griegos y sobre la tragedia no dejan la menor duda a este respecto: «La contradicción y el dolor son los únicos entes verdaderos» ${ }^{18}$, «la pluralidad está ya en la voluntad $»^{19}$ - se ve hasta qué punto, de manera fascinante, las nociones utilizadas en la ontología son contradichas en su acepción ordinaria en el momento mismo de su puesta en juego. El mismo estilo de escritura dando a entender una destitución y un desplazamiento de los esquemas mentales metafísicos clásicos se lee en esta otra definición: «la contradicción es la esencia de lo Uno originario» ${ }^{20}$. Si las fórmulas de «Uno originario» o incluso de «ser originario» no han dejado de fascinar a los comentaristas deseosos de reconciliar a toda costa a Nietzsche con el idealismo metafísico, es al precio desastroso de la «falta de filología» que ha sido mantenida por la reconciliación. Este «fundamento», este «Uno originario» deben entenderse, según las indicaciones de Nietzsche, en el sentido del dolor y la contradicción originarios, estas dos denominaciones son las más frecuentes en El nacimiento de la tragedia cuando se trata de referirse al fondo dionisíaco de la realidad.

Frente a las sorpresas y complicaciones del estudio en profundidad de El nacimiento de la tragedia, se puede estar tentado a volver a los primeros documentos que marcan la aparición de lo apolíneo y de lo dionisiaco en el corpus nietzscheano, con la esperanza de encontrar allí alguna luz, y de conocer cuál era la intención exacta de Nietzsche al experimentar la necesidad de elaborar estas nociones, acerca de las cuales una larga costumbre altera hoy quizás nuestra comprensión. Se sabe que es en el escrito del periodo de Basilea titulado La visión dionisiaca del mundo donde el filósofo introduce la pareja apolíneo/dionisíaco, y que hace de ella, simultáneamente, la clave interpretativa de la tragedia ática. Recuérdese igualmente que el texto fue redactado durante el verano de 1870, por lo tanto, poco antes de la versión final de El nacimiento de la tragedia ${ }^{21}$. De hecho, es extremamente instructivo comparar en particular la página que abre este opúsculo, que es como un primer esbozo de El nacimiento de la tragedia, con el muy célebre comienzo del parágrafo de esta última obra. Los dos procesos son introducidos allí desde el principio, como en la obra posterior, con la misma referencia a las divinidades del panteón griego, evocando igualmente los dos estados del sueño y la embriaguez. No se les atribuye, por el contrario, el estatus de "pulsiones», y son caracterizados como simples «antítesis estilísticas» (Stilgegensätze) ${ }^{22}$. La imagen de la diferencia de sexos tampoco aparece. Pero el punto esencial es que la explotación de los dos

\footnotetext{
18. FP I 198, KSA 7, 7 [169].

19. FP I 138, KSA 7, 5 [80].

20. FP I 194, KSA 7, 7 [157]

21. Sobre la situación del contexto y de la cronología de La visión dionisíaca del mundo, véase KSA 14, pp. 41 ss.

22. La visión dionisíaca del mundo, $\mathbb{S} 1$, OC I 461.
} 
modelos fisiológicos revela, en el detalle, divergencias sensibles con respecto a la lectura antitética, dualista, que constituye la interpretación usual (y quizás inadecuada, como se acaba de ver) de El nacimiento de la tragedia.

De entrada, hay un punto en el que merece la pena detenerse: al describir la lógica de la percepción apolínea de la apariencia como apariencia, Nietzsche, desde las primeras líneas de este escrito, hace la siguiente precisión: «También lo serio, triste, turbio y tenebroso es visto con el mismo placer, solo que también aquí el velo de la apariencia ha de estar en movimiento ondeante y no le es lícito cubrir por completo las formas fundamentales de lo real» ${ }^{23}$.

En principio, lo apolíneo se considera como algo secundario, en relación a lo que Nietzsche llama aquí, con una expresión que no se encuentra en El nacimiento de la tragedia, «las formas fundamentales de lo real» (die Grundformen des Wirklichen), y que parece entonces designar el mundo dionisíaco. Más arriba evocamos la extrañeza que suscita, al salir de El nacimiento de la tragedia, la lectura del $\$ 10$ de «Incursiones de un intempestivo» de Crepúsculo de los ídolos. Un vistazo a La visión dionisiaca del mundo desvanece esta sensación y nos muestra que no es seguro que haya verdaderamente una evolución de Nietzsche sobre la cuestión de la relación entre las dos pulsiones. Podríamos más bien tener la tentación de considerar que hay una especificidad de elección de presentación llevada a cabo en El nacimiento de la tragedia, y que es de ella de donde proceden las dificultades de comprensión.

Sin embargo, se puede sacar una primera conclusión: no hay aparentemente verdadera dicotomía entre las dos pulsiones, porque a fin de cuentas el mundo apolíneo de la apariencia se reduce siempre a la actividad de la pulsión dionisíaca. Nietzsche, quien denunciará tan vigorosamente y tan explícitamente el dualismo como uno de los prejuicios mayores de los filósofos, no habría por tanto defendido una posición diferente al elaborar su primera obra, contrariamente a lo que se podría suponer después de una rápida lectura. Y El nacimiento de la tragedia, exigiendo ya del lector ese arte del orfebre en el desciframiento que el prólogo añadido a Aurora elevará al rango de virtud cardinal de la filosofía, sería un libro con un doble fondo.

\section{3. ¿LO APOLÍNEO, CONDICIÓN DE POSIBILIDAD DE LO DIONISIACO?}

Incluso con un triple fondo. La situación, bien pensado, puede resultar todavía más complicada si uno se esfuerza en tener en cuenta todos los elementos que Nietzsche distribuye sabiamente a medida que se despliega la lógica que se desarrolla en el seno del texto de El nacimiento de la tragedia. Hemos visto que lo dionisiaco se encuentra a menudo como emboscado tras los análisis que elucidan el sentido de lo apolíneo. Pero ¿̇cómo se introduce lo dionisiaco en El nacimiento de la tragedia? Si se supera todo el primer parágrafo del primer capítulo, que, como se ha señalado, da la sensación de una simetría perfecta y, por lo tanto, un dualismo claro, se observa que en un cierto sentido es a partir de lo apolíneo como se opera esta entrada en escena; más exactamente, y esto siempre desde el primer capítulo, a partir de un debilitamiento de lo apolíneo, pero ciertamente no de su abolición completa: es a partir de la percepción de una «excepción» ${ }^{24}$ al

23. La visión dionisíaca del mundo, $\mathbb{S} 1$, OC I 461. El subrayado del final de la cita es nuestro.

24. El nacimiento de la tragedia, $\mathbb{\$} 1$. 
principio de razón, según la terminología schopenhaueriana, de una «ruptura del principium individuationis» 25 , «como si el velo de Maya estuviera roto y tan solo revolotease en jirones ante el misterioso Uno-primordial ${ }^{26}$, precisa Nietzsche; y el estado del hombre que sufre el furor dionisíaco se revela a través de una imagen: «en una imagen onírica de tipo metafórico» ${ }^{27}$. El velo se desgarra, pero no se suprime. De manera impresionante, el acceso a lo dionisíaco se juega a través de lo «onírico», de una persistencia del sueño.

Ahora estamos en un tercer tiempo, conducidos a admitir, o más bien constreñidos a admitir, que Dioniso ya no es pensable sin lo apolíneo, y que realmente no es identificable por separado. El punto determinante está en comprender que Nietzsche describe una lógica de la aparición, punto sobre el que James Porter ha sido, según nosotros, el primero en insistir con clarividencia. Insiste en ello, por ejemplo en el $\ 10$ de El nacimiento de la tragedia: «Pero, para servirnos de la terminología de Platón, se tendría que hablar de las figuras trágicas del escenario helénico poco más o menos de este modo: el único Dioniso verdaderamente real se manifiesta en una pluralidad de figuras, con la máscara de un héroe que lucha, y, por así decirlo, prisionero en la red de la voluntad individual. Tal y como ahora habla y actúa ese dios que se manifiesta, se asemeja a un individuo que comete errores, siente ansias y sufre: y el que se manifieste en general con esta precisión y esta claridad épicas es el efecto del Apolo intérprete de sueños, el cual mediante aquel fenómeno de tipo metafórico le interpreta al coro su estado dionisíaco ${ }^{28}$.

Se considera a Dioniso como un fenómeno de aparición gobernado por la autoridad de Apolo. Ciertamente, se podría objetar que esta afirmación concierne al contexto particular de la cooperación de dos pulsiones que constituyen la materia específica de la tragedia, por consiguiente de un momento de reconciliación y de cooperación, y que, por consiguiente, no se le puede otorgar el estatus de una afirmación con valor general. Pero en La visión dionisíaca del mundo, Nietzsche defiende sin equívoco, y esto en un sentido muy amplio, esta referencia del proceso dionisíaco mismo a la lógica — a primera vista absolutamente no dionisiaca- de la aparición. A propósito del actor, en tanto que hombre dionisíaco, indica que «no aspira a la bella apariencia, pero sí a la apariencia, no aspira a la verdad, pero sí a la verosimilitud ${ }^{29}$. Un par de páginas más adelante, coloca los dos procesos sobre el mismo plano, lo que significa esta vez despojar a lo dionisíaco de toda forma de fundamentación, y de toda lógica específica eludiendo aquella en la que se inscribe lo apolíneo: «ambas formas fenoménicas (Erscheinungsformen) de la voluntad» ${ }^{30}$. En tales textos, lo dionisiaco se ve reducido él también al movimiento constitutivo de la apariencia.

Y todavía más radicalmente, desde el principio de La visión dionisiaca del mun$d o$, Nietzsche asocia embriaguez y percepción de las apariencias como apariencias. En otros términos, mientras que El nacimiento de la tragedia opone, de la manera a primera vista más indiscutible, los dos fenómenos fisiológicos sirviendo de modelo

25. Ibid.

26. Ibid., $\mathbb{} 1$, OC I 341 .

27. Ibid., $\mathbb{\$} 2$, OC I 342 .

28. Ibid., $\mathbb{S} 10$, OC I 374 .

29. La visión dionisíaca del mundo, $\mathbb{\$} 3$, OC I 469. El subrayado del pasaje central es nuestro.

30. Ibid., $\$ 3$, OC I 471 . 
de inteligibilidad de las dos pulsiones de la naturaleza, La visión dionisiaca del mun$d o$, por el contrario, las aproxima, haciendo del sueño un modelo de inteligibilidad de la embriaguez. Se observa acertadamente un cambio de lógica. Retrospectivamente es algo sorprendente cómo es explícitamente analizado lo dionisíaco, incluso a partir del tipo de experiencia propia en el universo apolíneo, a partir de la lógica del sueño: «Este estado, si de él no hemos tenido experiencia en nosotros mismos, solo nos deja que lo captemos de una manera metafórica: es algo similar a cuando uno sueña y se está dando cuenta a la vez de que los sueños, sueños son ${ }^{31}$. Nietzsche trata por tanto de garantizar en 1870 al proceso dionisiaco el mismo tipo de estado y de dominio de sí persistente que hace, y continuará haciéndolo en su primer libro publicado, con respecto a lo apolíneo, el cual es caracterizado no solamente por el sueño, sino por una forma muy específica de sueño relativamente lúcida, o si se quiere de distanciamiento en el seno mismo del proceso onírico: «De este modo el servidor de Dioniso ha de estar en plena embriaguez y, al mismo tiempo, ha de estar al acecho detrás de sí mismo como observador. El rasgo distintivo de los artistas dionisíacos se muestra no en el cambio entre dominio de sí y embriaguez, sino en la coexistencia de ambos ${ }^{32}$. En otros términos, Nietzsche hace todo lo posible por evitar una oposición fuerte entre lo dionisiaco y lo apolíneo en el texto de 1870, hasta sugerir la reducción del primero al segundo.

Va todavía más lejos: mientras que los pasajes de El nacimiento de la tragedia que hemos señalado más arriba presuponen lo dionisiaco como basamento universal del fenómeno apolíneo, otros textos implican, al contrario, que este basamento correspondería más bien al proceso apolíneo mismo. De este modo, en La visión dionisiaca del mundo, la «voluntad», al menos la «voluntad helénica», parece ser comprendida a partir de una dimensión, o más bien bajo la forma de una orientación fundamentalmente apolínea: «Aquí se ha alcanzado el límite más peligroso que la voluntad helénica, con su principio apolíneo-optimista fundamental, podía permitir» ${ }^{33}$. Es la misma voluntad (en realidad la misma «voluntad»), según lo que afirma literalmente Nietzsche en estas líneas, la que limita el proceso dionisíaco, lejos de identificarse allí. ¿Podría ser, en estas condiciones, que El nacimiento de la tragedia sea un poco menos dionisiaco de lo que en general sugieren los comentaristas, que el lugar de lo apolíneo asuma mucho menos un papel secundario, incluso como figurante, como una opinión generalizada quiere? Cabe recordar que, aunque partidario de una lectura que equilibre el papel de las dos pulsiones, el fino lector que fue Walter A. Kaufmann ha invitado a los lectores a prestar la máxima atención a las sugerencias que implican de manera repetida un primado de lo apolíneo: «En El nacimiento de la tragedia Nietzsche no ensalzó a uno a expensas del otro, pero si favorece a uno de los dos dioses, este es Apolo» ${ }^{34}$.

¿Debe pensarse lo apolíneo, en estas condiciones, como la condición de posibilidad incluso de emergencia de lo dionisíaco? Una perspectiva semejante, como se ve, revoluciona de nuevo las seguridades que nosotros habíamos podido creer conseguidas después de la investigación que acabamos de realizar y ha conducido

31. Ibid., $\mathbb{S} 1$, OC I 462. Sobre esta cuestión véase los análisis de J. Porter, op. cit., pp. 53 s.

32. La visión dionisíaca del mundo, $\mathbb{\$} 1$, OC I 462.

33. Ibid., $\$ 3$, OC I 469.

34. W. A. Kaufmann, Nietzsche, Philosopher, Psychologist, Antichrist, Princeton, Princeton UP, 1950, reed. 1974, p. 128. 
a reconocer la universalidad de lo dionisíaco. La consistencia de lo real parece fundamentar, aligerarse, hasta evaporarse. Lo «metafísico» está condicionado por lo artístico - por lo ilusorio. Se accede al plano metafísico, al «subsuelo dionisíaco del mundo» ${ }^{35}, \mathrm{al}$ " fundamento de toda existencia» ${ }^{36}$, sometiéndose a la lógica apolínea de la apariencia ¿Qué estatuto ontológico se puede otorgar a este fundamento que solo es alcanzado por intermediación del mundo onírico, cuando incluso este último se rompe parcialmente? Así lo plantea muy pertinentemente James Porter, quien capta con exactitud esta lógica desconcertante: «¿En qué medida [...] lo dionisíaco es la invención de lo apolíneo?»37.

Lo apolíneo remite in fine a lo dionisiaco. Pero lo dionisiaco no se logra (o al menos no puede ser considerado) más que a partir de lo apolíneo. El nacimiento de la tragedia parece por tanto caracterizado por una situación de circularidad sorprendente, y ahí está quizás lo que quería pensar fundamentalmente Nietzsche en esta obra, presentando de esta manera una comprensión totalmente renovada de la realidad. Por primera vez, esta última sería disociada de la idea de objetividad, posición que Nietzsche sostendría cada vez más explícitamente en los años ochenta en particular, confiriendo a lo real el estatus de interpretación ${ }^{38}$. Y quizás esto era justamente lo que quería significar la ubicación del término Duplicität al comienzo de la obra. Así lo señala Celine Denat a propósito de este término difícil de comprender: «El 'carácter doble' de lo apolíneo y de lo dionisíaco no debe ser, por consiguiente, pensado como sinónimo de un simple dualismo: estamos tratando aquí no de una dicotomía de términos radicalmente independientes, sino de dos figuras de las que es preciso pensar, como lo confirmará a continuación el texto, la necesaria relación. La duplicidad de lo apolíneo y de lo dionisiaco tiene, en otras palabras, como contrapartida su complicidad, que se manifiesta plenamente en el artista y la obra trágica ${ }^{39}$. Es ciertamente en esta perspectiva en la que es preciso tratar de comprender la difícil fórmula «el misterio de su unidad», que utiliza Nietzsche en el $\mathbb{S}$ de El nacimiento de la tragedia: una situación que es un desafío para nuestra lógica dualista ordinaria, refundiendo carácter doble y unidad simultáneamente. Quizás Nietzsche ha querido, rechazando la simple noción de

35. El nacimiento de la tragedia, $\$ 25$, OC I 437.

36. Ibid.

37. J. I. Porter, The Invention of Dionysus. An Essay on The Birth of Tragedy, Stanford: Stanford UP, 2000, p. 49. Precisa: "The question is crucial, because it suggests something that cannot be countenanced on a reading of the Dionysian as the far extreme of any otherness, far beyond the banalities of the 'crude reality [plumpe Wirklichkeit]' that the Apollinian principle staves off». Véase igualmente el análisis desarrollado en las líneas siguientes: «The conditions of possibility of the Dionysian principle are located within the realm of appearances, possibly as one of their aspects (their appearances); they are not located somewhere else, in some autonomously and untouchably remote and metaphysically separable realm — for instance, in 'a sphere which is beyond and prior to all appearances', as the official version of Dionysian metaphysics has it ( $\mathbb{6} 6$; trans. mod.). I want to suggest that The Birth of Tragedy bears witness to the construction of such a beyond through the offices of Apollinian appearances. In other words, there is a pleat in the texture of appearance, and this pleat or fold in the skin of appearances is the constitutive factor of the metaphysical» (ibid.).

38. Sobre esta cuestión remitimos de nuevo a nuestro estudio «Oui, l'homme fut un essai», cit., y en particular el primer capítulo «La culture comme problème».

39. La naissance de la tragédie, trad. y presentación de C. Denat, Paris: GF-Flammarion, 2015, pp. 252-253. Véase igualmente la obra antes citada de James Porter, p. 42, quien sugiere también que en este contexto, «duplicidad» no puede identificarse con «dualidad». 
dualidad, procurar desde el comienzo que el lector sea consciente del hecho de que era una lógica totalmente nueva, la de una relación dual en la que cada uno de los polos remite al otro y lo presupone, que iba a establecerse para determinar de la manera más rigurosa el estatus exacto de lo real: una forma de ilusión, que no se ha de pensar como lo contrario de un ser o de una verdad, una ilusión artística —una transfiguración- que es el tejido mismo de nuestra relación con el mundo ${ }^{40}$. La ciencia misma, la reivindicación de la objetividad del proceso del conocimiento, es arte, y por lo tanto ilusión, como lo subraya el capítulo 15 de El nacimiento de la tragedia. La filosofía no hace excepción a la regla, ni aún menos la perspectiva filosófica que presenta El nacimiento de la tragedia, ella también produce una ilusión artística, como lo subraya el último capítulo de la obra. «Metafísica» muy atípica la de este primer libro que busca quizás hacernos comprender, en medio del juego de implicaciones recíprocas que despliega, que no es necesario hacer de lo dionisiaco un principio stricto sensu, en el sentido usual metafísico, una unidad absolutamente originaria que produciría todo el resto de lo real. Y que nuestra relación con lo real no es, en el universo que describe El nacimiento de la tragedia, jamás captada como neutra o reflejo de una objetividad, sino siempre deformación, reconfiguración, confirmando el inevitable primado de la ilusión.

[Traducción de José Honorio Ruiz Ortiz] $\underline{\text { ¿Universidad? }}$

40. Richard Schacht ha señalado que en el caso de lo apolíneo como en el de lo dionisíaco se da siempre un proceso de transfiguración : R. Schacht, Nietzsche, London : Routledge and Kegan Paul, 1985, p. 488-490. La diferencia entre las dos pulsiones tiende más bien a la variación de una lógica que gobierna la deformación que a una disparidad en el grado de la realidad objetiva a las que ellas serían susceptibles de dar acceso. 